\title{
Narrativa
}

narrativa

Nuova serie

$43 \mid 2021$

La fantascienza nelle narrazioni italiane ipercontemporanee

\section{Ugo FRACASSA (a cura di), Moti di imitazione. Teorie della mimesi e letteratura}

Maria Luisa Sais

\section{OpenEdition}

1 Journals

Edizione digitale

URL: https://journals.openedition.org/narrativa/476

DOI: $10.4000 /$ narrativa.476

ISSN: 2804-1224

Editore

Presses universitaires de Paris Nanterre

\section{Edizione cartacea}

Data di pubblicazione: 1 décembre 2021

Paginazione: 296-297

ISBN: 978-2-84016-485-2

ISSN: $1166-3243$

\section{Notizia bibliografica digitale}

Maria Luisa Sais, «Ugo fracASSA (a cura di), Moti di imitazione. Teorie della mimesi e letteratura», Narrativa [Online], 43 | 2021, online dal 01 novembre 2021, consultato il 03 novembre 2022. URL: http://

journals.openedition.org/narrativa/476 ; DOI: https://doi.org/10.4000/narrativa.476

Questo documento è stato generato automaticamente il 3 novembre 2022.

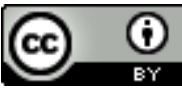

Creative Commons - Attribuzione 4.0 Internazionale - CC BY 4.0

https://creativecommons.org/licenses/by/4.0/ 


\title{
Ugo FRACASSA (a cura di), Moti di imitazione. Teorie della mimesi e letteratura
}

\author{
Maria Luisa Sais
}

\section{NOTIZIA}

Ugo FRACASSA (a cura di), Moti di imitazione. Teorie della mimesi e letteratura, Milano, Morellini, 2020, $200 \mathrm{p}$.

1 Le teorie della mimesi, o imitazione, conoscono nuova linfa nella seconda metà del secolo scorso, a partire dalla pubblicazione dell'emblematico saggio di René Girard, Menzogna romantica e verità romanzesca (1961), in cui il critico indaga la natura del desiderio nel romanzo europeo sottolineandone il carattere imitativo: "il desiderio non è mai spontaneo, ma condizionato". La linea del desiderio sarebbe dunque triangolare, andando a includere, tra un soggetto desiderante e l'oggetto del desiderio, un mediatore che è poi il vero fine a cui tende il primo termine di paragone. Desiderio è desiderio di essere l'altro, di impossessarsi della sua essenza e dei suoi desideri, non appropriarsi di ciò che ha, in uno schema antropologico in cui l'imitazione del modello gioca un ruolo fondamentale. Quasi a conferma di ciò che è stato elaborato in ambito umanistico, arriva, a metà degli anni Novanta, la scoperta rivoluzionaria dei cosiddetti neuroni specchio, che, oltre ad aprire nuovi scenari sul funzionamento del cervello e sui meccanismi dell'empatia e dell'imitazione, incentiva il dialogo interdisciplinare, un confronto fruttuoso tra i saperi basato sulla "nozione-ponte" di mimesi. Il volume curato da Ugo Fracassa ben interpreta questo sodalizio fra vari ambiti conoscitivi, dimostrando l'estrema malleabilità di un concetto che vanta secoli di elaborazione teorica e filosofica, quello di mimesis appunto, e riservando un posto privilegiato alla letteratura, anche in virtù del fatto che i suoi studiosi si siano "tra i primi e con maggiore profitto, disposti a un dialogo con le neuroscienze". 
2 La prima parte del saggio raccoglie degli interventi che spaziano dalla letteratura alla filosofia, dalla psicologia alle neuroscienze, proponendo ciascuno la propria teoria mimetica, in alcuni casi in contraddizione con le altre. Per Cimatti, ad esempio, "la soggettività è un punto di arrivo, non di partenza". Quando si nasce non si ha nessuna percezione di sé, si è invece inseriti all'interno di un "campo sociale" in cui agiscono delle forze - $\mathrm{i}$ "moti di imitazione" del titolo - che spingono il singolo a voler essere come l'altro. A partire da una tale premessa, l'obiettivo è quello di fornire una risposta a uno dei quesiti più spinosi della filosofia novecentesca: come si forma l'individualità autonoma in un mondo dominato da forze centrifughe? Come si passa dall'imitazione alla creatività? Mormino riprende studi e premesse dell'articolo precedente per arrivare a tutt'altre conclusioni: il rapporto con gli altri sarebbe secondario rispetto alla percezione che l'individuo ha di sé, l'imitazione semplicemente "un'illusione ottica". Imitare gli altri equivale, paradossalmente, ad "autoimitarsi". Altre interessanti riflessioni di questa prima metà del libro riguardano i "moti di deformazione" - alla base del lavoro del caricaturista, il quale ha come fine ultimo non una rappresentazione oggettiva della realtà, ma l'esasperazione dei dettagli, ricalcando il meccanismo conoscitivo della nostra mente - e l'embodiment, il coinvolgimento del corpo nella creazione dell'esperienza estetica.

La seconda parte del volume propone invece degli articoli in cui le teorie mimetiche vengono applicate ai testi letterari. Singolare è l'operazione svolta da Simonetti, il quale dimostra come la teoria mimetica di Girard possa essere utilizzata non solo per architettare la struttura di un romanzo, ma anche per spiegare il rapporto "triangolare" tra due autori, Siti e Pasolini, in cui il secondo risulta essere mediatoremodello, ma anche bersaglio del primo. "L'angoscia dell'influenza" (si veda Bloom nel primo articolo di Tagliaferri) porta Siti a riscrivere, emendare, rovesciare Pasolini, tanto che la sua intera opera può essere letta alla luce della produzione intellettuale di quest'ultimo. La crisi d'identità dell'uomo moderno, che tende a costruire di sé un'immagine idealizzata difficilmente raggiungibile rispecchiandosi nello sguardo altrui, consente invece di azzardare l'accostamento, proposto nel saggio di Tinelli, tra Dostoevskij e Carrère, testimoni entrambi dell'isolamento dell'individuo che vive delle proprie fantasie e misconosce la realtà. Il volume si conclude con le analisi dei testi di Wallace (Scarlato) e Unamuno (Liuzzo): il saggio di Scarlato permette di riflettere sulla relazione che intercorre tra imitazione ed empatia, sulle dinamiche di identificazione che l'autore deve attivare se vuole "toccare le persone" attraverso la letteratura (p. 157); quello di Liuzzo fornisce un'ultima e significativa esemplificazione letteraria dell'impianto teoretico girardiano. 